\title{
EXCEPTIONAL POINTS FOR COCOMPACT FUCHSIAN GROUPS
}

\author{
Joseph Fera \\ Lehman College CUNY, Department of Mathematics and Computer Science \\ 250 Bedford Park Blvd West, Bronx, NY 10468, U.S.A.; joseph.fera@lehman.cuny.edu
}

\begin{abstract}
Let $G$ be a cocompact Fuchsian group acting on the hyperbolic plane $\mathbf{H}$. If $G$ covers a compact hyperbolic surface of genus $g \geq 2$, then almost every Dirichlet region for $G$ has $12 g-6$ sides. In this article, we study the exceptional points for $G$, i.e., the points in $\mathbf{H}$ associated to Dirichlet regions for $G$ with strictly less than $12 g-6$ sides. More specifically, we show that uncountably many exceptional points exist for any cocompact group. We also define and prove the existence of higher order exceptional points for any such group.
\end{abstract}

\section{Introduction}

We consider cocompact Fuchsian groups $G$ that cover compact hyperbolic surfaces of genus $g \geq 2$. For such groups, Beardon proved that almost every Dirichlet region has $12 g-6$ sides [1]. Any point $z$ in the hyperbolic plane $\mathbf{H}$ that admits a Dirichlet region for $G$ with strictly less than $12 g-6$ sides is called exceptional for $G$. As a consequence of Beardon's result, the set of exceptional points for any cocompact $G$ has zero measure and, a priori, may be empty. In what follows, we prove the following result using topological results on a Dirichlet region's side-pairing transformations.

Theorem 4.3. Let $G$ be a cocompact Fuchsian group. Uncountably many exceptional points exist for $G$ in $\mathbf{H}$.

To better understand exceptional points, we also investigate where in $\mathbf{H}$ such points can exist. We call these locations indicating sets for $G$. They appear in [5] and are defined via complex-valued rational functions and hyperbolic isometries. We provide useful topological characterizations for indicating sets (Section 5), which allow us to prove an existence result for Dirichlet regions with strictly less than $12 g-8$ sides. Points admitting such Dirichlet regions are called exceptional points of higher order for $G$.

Theorem 6.3. If $G$ is a cocompact Fuchsian group, then there exists an exceptional point of higher order for $G$.

The paper proceeds as follows. Section 2 contains the definitions, notations, and foundational results on cocompact Fuchsian groups needed for subsequent sections. It also includes several technical facts (with minimal proofs) on Dirichlet regions. We refer to [1], [3], and [4] for this background information. Section 3 summarizes known results on regular points, i.e., the points in $\mathbf{H}$ that admit Dirichlet regions with $12 g-6$ sides. In Section 4, we prove Theorem 4.3. Section 5 investigates indicating

doi:10.5186/aasfm.2014.3917

2010 Mathematics Subject Classification: Primary 20H10; Secondary 30F10.

Key words: Cocompact Fuchsian groups, Dirichlet regions. 
sets and provides a key proposition used to prove Theorem 6.3 which is the content of Section 6.

\section{Preliminaries}

Let $\mathbf{H}$ denote the hyperbolic plane, $\rho$ the hyperbolic metric on $\mathbf{H}$, and $\operatorname{Isom}^{+}(\mathbf{H})$ the group of all orientation-preserving hyperbolic isometries endowed with the compact-open topology. A discrete subgroup $G$ of $\operatorname{Isom}^{+}(\mathbf{H})$ is called a Fuchsian group. Unless otherwise stated, $G$ denotes a finitely generated, fixed-point free Fuchsian group. When given the quotient topology, the quotient space $\mathbf{H} / G$ is homeomorphic to a sphere with $g \geq 2$ many handles and finitely many punctures. This $g$ is the genus of $\mathbf{H} / G$.

The quotient space $S=\mathbf{H} / G$ is a hyperbolic surface and inherits a complete hyperbolic metric denoted by $\rho_{S}$. Recall that a fundamental set for the action of a Fuchsian group $G$ on $\mathbf{H}$ is a subset $F$ of $\mathbf{H}$ that contains exactly one point from each $G$-orbit. An open and connected subset $D$ of $\mathbf{H}$ is called a fundamental domain for $G$ provided that the hyperbolic area of $\partial D$ is 0 and there exists a fundamental set $F$ for $G$ that that satisfies $D \subset F \subset \bar{D}$, where $\bar{D}$ denotes the closure of $D$. The following construction defines a family of fundamental domains, the so-called Dirichlet regions, for the action of such $G$ on $\mathbf{H}$.

Definition 2.1. The Dirichlet region for $G$ with basepoint $z$ is defined by,

$$
D(z)=\bigcap_{f \in G \backslash\{\mathrm{id}\}} H_{f}(z),
$$

where $H_{f}(z)=\{w \in \mathbf{H}: \rho(w, z)<\rho(w, f(z))\}$.

When the hyperbolic surface $\mathbf{H} / G$ is compact, the Fuchsian group $G$ is called cocompact. Such groups are necessarily of the first kind (i.e. their limit sets are all of $\partial \mathbf{H})$ and contain only hyperbolic isometries. Therefore, all such groups are necessarily torsion-free. In addition, if $G$ is cocompact, then any of its associated Dirichlet regions is a finite-sided hyperbolic polygon with compact closure, $\overline{D(z)}$, in H.

A side $s$ of $D(z)$ is a positive length geodesic segment of the form $\overline{D(z)} \cap f(\overline{D(z)})$ where $f \in G \backslash\{\mathrm{id}\}$. A vertex of $D(z)$ is a single point of the form $\overline{D(z)} \cap f(\overline{D(z)}) \cap$ $h(\overline{D(z)})$ where $f, h \in G \backslash\{\operatorname{id}\}$. For each side $s$ of $D(z)$, there exists a unique map $f \in G$ such that $f(s)$ is another side of $D(z)$. The collection of all such maps, called the side-pairing transformations of $D(z)$, form a subset of $G$ denoted by $S(z)$. Since $G$ is torsion-free, it follows that the number of side-pairing transformations for $D(z)$, given by $|S(z)|$, equals the number of sides of $D(z)$.

The following result, due to Beardon [1, Theorem 10.1.2], provides both upper and lower bounds on $|S(z)|$ for cocompact $G$.

Theorem 2.2. Let $G$ be a cocompact Fuchsian group such that $\mathbf{H} / G$ has genus $g \geq 2$. Then,

$$
4 g \leq|S(z)| \leq 12 g-6 .
$$

and the upper bound is attained on a full-measure subset of $\mathbf{H}$.

In what follows, $G$ will always denote a cocompact Fuchsian group and $D(z)$ the Dirichlet region of $G$ with center $z \in \mathbf{H}$. 
A cycle $C$ in $\overline{D(z)}$ is the intersection of the $G$-orbit of any point in $\mathbf{H}$ with $\overline{D(z)}$. Any cycle $C$ is necessarily finite by discreteness; its length is denoted by $|C|$. The next theorem combines consequences of Theorems 9.3.5 and 9.4.5 in [1].

Theorem 2.3. Let $C$ be a cycle in $\overline{D(z)}$ containing $w$. Then,

(1) $|C|=1$ if and only if $w \in D(z)$;

(2) $|C|=2$ if and only if $w$ is an interior point of a side of $D(z)$;

(3) $|C| \geq 3$ if and only if $w$ is a vertex of $D(z)$; such cycles are referred to as a vertex cycles of $D(z)$.

Next, we record several facts as one proposition for later reference. Their proofs follow directly from results in Section 9 of [1].

Proposition 2.4. Let $G$ denote a cocompact Fuchsian group and suppose that $f \in G$.

(1) Let $s$ be a side of $D(z)$. The side $s$ is given by $\overline{D(z)} \cap f(\overline{D(z)})$ if and only if $s \subset L_{f}(z)$, where

$$
L_{f}(z)=\{w \in \mathbf{H}: \rho(w, z)=\rho(w, f(z))\} .
$$

(2) The element $f$ is in $S(z)$ if and only if $D(z)$ has a side given by $s=\overline{D(z)} \cap$ $f^{-1}(\overline{D(z)})$.

(3) If $f \in S(z)$, then $f^{-1} \in S(z)$.

(4) If both $w$ and $f^{-1}(w)$ belong to the same cycle $C$ of $D(z)$, then

$$
\rho(w, z)=\rho\left(f^{-1}(w), z\right)=\rho(w, f(z)) .
$$

The lemma below is easily verified; it will be referenced in the proposition that immediately follows it and in later sections.

Lemma 2.5. If $A \subset \mathbf{H}$ is compact, then there exists $K \subset \mathbf{H}$ compact so that $\overline{D(z)} \subset K$ for all $z \in A$.

The following proposition is probably known, but we include a full proof for the reader's convenience. Throughout, $B_{R}(z)$ denotes the hyperbolic ball centered at $z \in \mathbf{H}$ of radius $R>0$. Its closure is denoted by $\overline{B_{R}(z)}$.

Proposition 2.6. If $z_{n} \in \mathbf{H}$ converge to $z \in \mathbf{H}$ in the hyperbolic metric, then $\partial D\left(z_{n}\right)$ converge to $\partial D(z)$ in the Hausdorff topology.

Proof. We show that for each $\varepsilon>0$ there exists $N \in \mathbf{N}$ such that for all $n \geq N$ the following conditions hold: (i) $\rho(w, \partial D(z))<\varepsilon$ for all $w \in \partial D\left(z_{n}\right)$ and (ii) $\rho\left(w, \partial D\left(z_{n}\right)\right)<\varepsilon$ for all $w \in \partial D(z)$. Using Lemma 2.5 with $A=\left\{z_{n}: n \in \mathbf{N}\right\} \cup\{z\}$ which is compact by the convergence assumption, we can find a compact set $K$ containing the collection of all $\overline{D\left(z_{n}\right)}$ and $\overline{D(z)}$.

Suppose to the contrary that (i) fails. Then, there exists an $\varepsilon>0$, an infinite subsequence of $\left\{z_{n}\right\}$ relabeled $\left\{z_{n}\right\}$ for convenience, and corresponding points $\zeta_{n} \in$ $\partial D\left(z_{n}\right)$ satisfying, $\rho\left(\zeta_{n}, \partial D(z)\right) \geq \varepsilon$ for every $n$. Each $\zeta_{n}$ lies in the compact set $K$ and, hence, a subsequence $\left\{\zeta_{n}\right\}$ relabeled if necessary may be extracted such that $\left\{\zeta_{n}\right\}$ converges to $\zeta \in K$. The limit $\zeta$ clearly satisfies $\rho(\zeta, \partial D(z)) \geq \varepsilon$.

For each $\zeta_{n}$, there exists $f_{n} \in G$ such that $\zeta_{n} \in L_{f_{n}}\left(z_{n}\right)$ by Proposition 2.4. It follows immediately that $\rho\left(z_{n}, \zeta_{n}\right)=\rho\left(f_{n}\left(z_{n}\right), \zeta_{n}\right)$ for every $n$. The compact set $K$ has 
finite diameter, say $M>0$, and $K$ contains all $z_{n}$ and $\zeta_{n}$. Therefore, $\rho\left(f_{n}\left(z_{n}\right), \zeta_{n}\right)=$ $\rho\left(z_{n}, \zeta_{n}\right)<M$ and we have $\rho\left(z_{n}, f_{n}\left(z_{n}\right)\right)<2 M$ by the triangle inequality.

If $K^{*}=\overline{B_{2 M}(K)}$, then $K^{*}$ is a compact set containing all $z_{n}$ and all $f_{n}\left(z_{n}\right)$. Hence, $f_{n}\left(K^{*}\right) \cap K^{*} \neq \emptyset$ for each $n$. Since $G$ is discrete, it follows that only finitely distinct maps exist among the $f_{n}$. So, there exists a map $f \in G$ such that $f_{n}=f$ for infinitely many $n \in \mathbf{N}$.

Again, refine and relabel the subsequence $\left\{\zeta_{n}\right\}$ so that, for all $n, \zeta_{n} \in L_{f}\left(z_{n}\right)$ for this fixed $f \in G$. For each $n, \rho\left(z_{n}, \zeta_{n}\right)=\rho\left(f\left(z_{n}\right), \zeta_{n}\right)$, with $z_{n} \rightarrow z$ and $\zeta_{n} \rightarrow \zeta$. It follows, from the continuity of $\rho$, that $\rho(z, \zeta)=\rho(f(z), \zeta)$ and, hence, $\zeta \in L_{f}(z)$. One now checks that $\zeta \in \partial D(z)$ which contradicts the fact that $\rho(\zeta, \partial D(z)) \geq \varepsilon$.

Next, we need to prove (ii). Suppose to the contrary that (ii) fails. Then, there exists $\varepsilon>0$ and a collection of points $\zeta_{n} \in \partial D(z)$ such that $\rho\left(\zeta_{n}, \partial D\left(z_{n}\right)\right) \geq \varepsilon$ for infinitely many $n$. Since $D(z)$ is a finite-sided polygon with compact closure in $\mathbf{H}$, its boundary is compact and, hence, the collection of $\zeta_{n} \in \partial D(z)$ has at least one accumulation point $\zeta \in \partial D(z)$.

We claim that $\zeta$ satisfies $\rho\left(\zeta, \partial D\left(z_{n}\right)\right) \geq \varepsilon / 2$ for large enough values of $n$. Observe that $\rho\left(\zeta_{n}, \partial D\left(z_{n}\right)\right) \geq \varepsilon$ by assumption, and for large enough $n$ we have $\rho\left(\zeta_{n}, \zeta\right)<\varepsilon / 2$. Thus, by the (reverse) triangle inequality, $\rho\left(\zeta, \partial D\left(z_{n}\right)\right) \geq \varepsilon / 2$ for these large enough $n$. It follows that, for these $n$, there exists an open neighborhood $U$ of $\zeta$ such that either $U \subset D\left(z_{n}\right)$ or $U \subset \mathbf{H} \backslash \overline{D\left(z_{n}\right)}$. It is not hard to verify that neither can happen for infinitely many $n \in \mathbf{N}$ which results in a contradiction and shows (ii).

\section{Regular points}

Recall that $G$ denotes a cocompact Fuchsian group, $D(z)$ the Dirichlet region for $G$ centered at $z$, and $S(z)$ the collection of all side-pairing transformations of $D(z)$.

Proposition 3.1. Let $z \in \mathbf{H}$ and $f \in S(z)$. Then there exists an open set $U \subset \mathbf{H}$ containing $z$ such that $f \in S(w)$ for all $w \in U$.

Proof. Suppose to the contrary that the statement is false. Hence, there exists a sequence $\left\{z_{m}\right\}$ converging to $z$ such that $f \notin S\left(z_{m}\right)$ for each $m \in \mathbf{N}$. It follows from Proposition 2.4 that $D\left(z_{m}\right)$ cannot have a side of the form $s_{m}=\overline{D\left(z_{m}\right)} \cap f\left(\overline{D\left(z_{m}\right)}\right)$ for any $m \in \mathbf{N}$. We proceed to show, however, that this cannot be the case for infinitely many $s_{m}$.

Since $f \in S(z)$, the Dirichlet region $D(z)$ has a side given by $s=\overline{D(z)} \cap$ $f(\overline{D(z)}) \subset L_{f}(z)$. Let $w$ be a point on $s$ that is not a vertex of $D(z)$. By Theorem 2.6, we can find points $w_{m} \in \partial D\left(z_{m}\right)$ such that $w_{m} \rightarrow w$ in the hyperbolic metric. Moreover, since a fixed Dirichlet region has only finitely many vertices, we may choose the $w_{m}$ so that no $w_{m}$ corresponds to a vertex of $D\left(z_{m}\right)$. Hence, $w_{m}$ is an interior point of some side $s_{m}$ of $D\left(z_{m}\right)$. By Theorem 2.3, there exists a unique map $h_{m}^{-1}$ so that $\left\{w_{m}, h_{m}^{-1}\left(w_{m}\right)\right\}$ defines the full cycle containing $w_{m}$. In addition, $\rho\left(w_{m}, z_{m}\right)=\rho\left(h_{m}^{-1}\left(w_{m}\right), z_{m}\right)$ by Proposition 2.4.

Choose, by Lemma 2.5 , a compact set $K$ containing each $\overline{D\left(z_{m}\right)}$ and $\overline{D(z)}$. Observe that $\left\{w_{m}, h_{m}^{-1}\left(w_{m}\right)\right\} \subset \overline{D\left(z_{m}\right)} \subset K$ for each $m$. Hence, $K \cap h_{m}^{-1}(K) \neq \emptyset$ for each $m$. Using the discreteness of $G$ we find that there exists $h \in G$ such that $h_{m}=h$ for infinitely many $m$. 
By refining our subsequence and relabeling if necessary, we may thus find an infinite collection of points $w_{m}$ which satisfy $\rho\left(w_{m}, z_{m}\right)=\rho\left(h^{-1}\left(w_{m}\right), z_{m}\right)$ for each $m$. By taking $m \rightarrow \infty$, it follows that $\rho(w, z)=\rho(w, h(z))$. Thus, $w \in L_{h}(z)$. Since $w$ is an interior point of $s \subset L_{f}(z)$, it must be the case that $L_{h}(z)=L_{f}(z)$; else, $w \in L_{f}(z) \cap L_{h}(z)$ would be a vertex of $D(z)$. As concluded previously, this implies that $h=f, \rho\left(w_{m}, z_{m}\right)=\rho\left(w_{m}, f\left(z_{m}\right)\right)$, and $w_{m} \in L_{f}\left(z_{m}\right)$. Since $w_{m}$ was chosen to lie on the interior of side $s_{m}$, it follows, by Proposition 2.4, that $s_{m}=\overline{D\left(z_{m}\right)} \cap f\left(\overline{D\left(z_{m}\right)}\right)$ for each $m$ and this is a contradiction.

For the remainder of this section, let $g$ be the genus of $G$ (note that we assume $G$ to be cocompact). Recall that, for almost every $z \in \mathbf{H}$, the associated Dirichlet region satisfies $|S(z)|=12 g-6$ by Theorem 2.2 .

Definition 3.2. A point $z \in \mathbf{H}$ is called regular for $G$ provided that $|S(z)|=$ $12 g-6$. If $z$ is not regular for $G$, then it is called exceptional for $G$.

Proposition 3.3. If $z \in \mathbf{H}$ is regular for $G$, then there exists an open set $U$ containing $z$ such that $S(u)=S(z)$ for all $u \in U$.

Proof. Let $z \in \mathbf{H}$ be regular for $G$ with $S(z)=\left\{f_{1}, f_{2}, \ldots, f_{12 g-6}\right\}$. For each $i \in\{1,2, \ldots, 12 g-6\}$, there exists an open set $U_{i} \subset \mathbf{H}$ with $z \in U_{i}$ such that $f_{i} \in S(w)$ for all $w \in U_{i}$; this follows from Theorem 3.1. Define,

$$
U=\bigcap_{i=1}^{12 g-6} U_{i} .
$$

By construction, $U$ is an open subset of $\mathbf{H}$ containing $z$ such that $S(z) \subset S(u)$ for all $u \in U$. Hence $12 g-6=|S(z)| \leq|S(u)| \leq 12 g-6$ for all $u \in U$. Hence, $S(u)=S(z)$ for all $u \in U$ and this completes the proof.

The following theorem is immediate.

Theorem 3.4. The set of all regular points for $G$ is an open subset of $\mathbf{H}$.

\section{Exceptional points exist}

Theorem 2.2 indicates that the set of all exceptional points for $G$ has zero measure in $\mathbf{H}$. In what follows, we prove that this zero-measure subset is non-empty. In fact, we prove that it is uncountable.

The following lemma is a standard result for non-elementary (which includes cocompact) Fuchsian groups [3].

Lemma 4.1. For any $z \in \mathbf{H}$ and any $\zeta \in \partial \mathbf{H}$, there exists $f \in S(z)$ so that $f(\zeta) \neq \zeta$.

The next result is known, but we include a proof for completeness.

Theorem 4.2. Fix $z_{0} \in \mathbf{H}$. The collection $\left\{z \in \mathbf{H}: S(z)=S\left(z_{0}\right)\right\}$ is bounded in the hyperbolic metric.

Proof. Assume to the contrary that $\left\{z \in \mathbf{H}: S(z)=S\left(z_{0}\right)\right\}$ is unbounded in the hyperbolic metric. Hence, there exists a sequence $\left\{z_{n}\right\} \subset \mathbf{H}$ with $S\left(z_{n}\right)=S\left(z_{0}\right)$ for all $n \in \mathbf{N}$ satisfying $\rho\left(z_{0}, z_{n}\right) \rightarrow \infty$ as $n \rightarrow \infty$. The closure of $\mathbf{H}$, denoted $\overline{\mathbf{H}}$, is compact in $\widehat{\mathbf{C}}$. Therefore, we can extract a convergent subsequence (in the chordal metric) from the collection $\left\{z_{n}\right\}$ and by relabeling if necessary we may assume that 
$z_{n} \rightarrow \zeta \in \overline{\mathbf{H}}$. Since $\rho\left(z_{0}, z_{n}\right) \rightarrow \infty$ as $n \rightarrow \infty$, it follows that $\zeta \in \partial \mathbf{H}$. Lemma 4.1 asserts the existence of $f \in S\left(z_{0}\right)$ so that $f(\zeta) \neq \zeta$. We proceed to show that $f \notin S\left(z_{n}\right)$ for large enough values of $n$, which results in a contradiction.

The hyperbolic surface $S=\mathbf{H} / G$ is compact by assumption and, hence, has finite diameter $M>0$. Thus, $D(z) \subset \overline{B_{M}(z)}$ for all $z \in \mathbf{H}$. By assumption, the group $G$ is cocompact implying that the map $f \in S\left(z_{0}\right) \subset G$ is hyperbolic and satsfies,

$$
\sinh \left(\frac{1}{2} \rho(z, f(z))\right)=\cosh \left(\rho\left(z, A_{f}\right)\right) \sinh \left(\frac{1}{2} \ell(f)\right),
$$

for all $z \in \mathbf{H}$. We note that $A_{f}$ and $\ell(f)$ denote the axis and translation length of $f$ respectively and refer to $[1$, Theorem 7.35]. The map $f$ does not fix $\zeta \in \mathbf{H}$. Hence, its axis $A_{f}$ does not intersect $\partial \mathbf{H}$ at $\zeta$ and so, $\rho\left(z_{n}, A_{f}\right) \rightarrow \infty$ as $n \rightarrow \infty$. It now follows from Equation (4.1) that $\rho\left(z_{n}, f\left(z_{n}\right)\right) \rightarrow \infty$ and thus for large enough $n, \rho(w, f(w))>3 M$ for all $w \in \overline{B_{M}\left(z_{n}\right)}$. But, $f \in S\left(z_{n}\right)$ for all $n$, and so, there exists $w \in \mathbf{H}$ such that $w, f(w) \in \partial D\left(z_{n}\right)$. Since $\overline{D\left(z_{n}\right)} \subset \overline{B_{M}\left(z_{n}\right)}$, we find that $\rho(w, f(w)) \leq 2 M$ which is a contradiction.

Theorem 4.3. Let $G$ be a cocompact Fuchsian group. Uncountably many exceptional points exist for $G$ in $\mathbf{H}$.

Proof. Throughout this proof, we work in the Poincaré disk model, $\mathbf{D}$, of $\mathbf{H}$. The set of regular points for $G$ is of full measure and, hence, by conjugation (if necessary) we may assume that 0 is regular for $G$. By Theorem 4.2 , the set $\{z \in$ $\mathbf{H}: S(z)=S(0)\}$ is bounded. Hence, there exists $M>0$ so that if $\rho(0, z)>M$, then $S(z) \neq S(0)$. Thus, for each $\theta \in[0,2 \pi)$, there exists $z_{\theta}=r_{\theta} e^{i \theta}$ with $0<r_{\theta}<1$ such that $S\left(z_{\theta}\right) \neq S(0)$. Let $\gamma_{\theta}$ denote the geodesic segment connecting 0 and $z_{\theta}$. We claim that, for each $\theta \in[0,2 \pi), \gamma_{\theta}$ contains an exceptional point for $G$.

Suppose to the contrary that there exists $\theta \in[0,2 \pi)$ so that $\gamma_{\theta}$ contains no exceptional point for $G$. Using the regular points along $\gamma_{\theta}$ and their associated open sets given by Proposition 3.3, we may create an open cover for $\gamma_{\theta}$ which, by compactness, has an associated finite subcover. Standard arguments now establish that $S(0)=S\left(z_{\theta}\right)$ contradicting the choice of $z_{\theta}$. So, $\gamma_{\theta}$ must contain an exceptional point for each $\theta \in[0,2 \pi)$.

Theorem 4.3 proves that, for any cocompact group $G$, the zero-measure subset of $\mathbf{H}$ of exceptional points for $G$ is non-empty and uncountable. Since Fuchsian groups are at most countable, see [3], it also shows that the set of exceptional points for $G$ contains representatives from uncountably many distinct $G$-orbits. Hence, if $\pi: \mathbf{H} \rightarrow \mathbf{H} / G$ denotes the canonical projection map, then there exists uncountably many $p_{j} \in \mathbf{H} / G$ such that each point in $\pi^{-1}\left(p_{j}\right) \subset \mathbf{H}$ is exceptional for $G$.

\section{Indicating sets}

In the proof of [1, Theorem 10.5.1], it is shown that $D(z)$ has less than $12 g-6$ if and only if there exists a boundary cycle $C$ of $D(z)$ of length at least 4 . Therefore, if $z$ is exceptional for $G$, then there exist distinct, non-identity maps $f_{1}^{-1}, f_{2}^{-1}, f_{3}^{-1} \in G$ and $w \in \partial D(z)$ such that $\left\{w, f_{1}^{-1}(w), f_{2}^{-1}(w), f_{3}^{-1}(w)\right\}$ belong to a boundary cycle $C$ of $D(z)$. It follows from Proposition 2.4, that

$$
\rho(z, w)=\rho\left(z, f_{1}^{-1}(w)\right)=\rho\left(z, f_{2}^{-1}(w)\right)=\rho\left(z, f_{2}^{-1}(w)\right) .
$$


Since each $f_{j}$ is a hyperbolic isometry, (5.1) is equivalent to

$$
\rho(w, z)=\rho\left(w, f_{1}(z)\right)=\rho\left(w, f_{2}(z)\right)=\rho\left(w, f_{3}(z)\right) .
$$

Equation (5.2) indicates that the four points $z, f_{1}(z), f_{2}(z)$, and $f_{3}(z)$ lie on a (hyperbolic) circle centered at $w$ in $\mathbf{H}$. We record this fact in the following proposition which can be found in [1, Theorem 9.4.5].

Proposition 5.1. Let $G$ be a cocompact Fuchsian group. If $z$ is exceptional for $G$, then there exist distinct, non-identity maps $f_{1}, f_{2}, f_{3} \in G$ such that,

$$
\operatorname{Im}\left(\frac{\left(z-f_{2}(z)\right)\left(f_{1}(z)-f_{3}(z)\right)}{\left(z-f_{1}(z)\right)\left(f_{2}(z)-f_{3}(z)\right)}\right)=0 .
$$

Equivalently, the complex cross-ratio $\left[z, f_{1}(z), f_{2}(z), f_{3}(z)\right]$ is purely real.

Note that the collection of points in $\widehat{\mathbf{C}}$ satisfying (5.3) lies on $R^{-1}(\widehat{\mathbf{R}})$ where $R$ is the rational map given by,

$$
R(z)=\frac{\left(z-f_{2}(z)\right)\left(f_{1}(z)-f_{3}(z)\right)}{\left(z-f_{1}(z)\right)\left(f_{2}(z)-f_{3}(z)\right)}
$$

for distinct, non-identity maps $f_{1}, f_{2}, f_{3} \in G$. These rational maps will help to characterize the possible locations of exceptional points for $G$.

Definition 5.2. For distinct, non-identity maps $f_{1}, f_{2}, f_{3} \in G$, let $R(z)$ be defined as in (5.4). Then, the set $\Gamma=R^{-1}(\widehat{\mathbf{R}}) \cap \mathbf{H}$ is called an indicating set for G.

By Proposition 5.1, if $z$ is exceptional for $G$, then there exists an indicating set $\Gamma$ for $G$ such that $z \in \Gamma$. The converse of this statement, however, does not hold in general. Though she uses different terminology, Näätänen studies the indicating sets of a fixed cocompact Fuchsian group in $\S 3$ of [5]. Her results imply that not all points found in these sets are exceptional.

Since $G$ has at most countably many elements, the collection of all indicating sets for $G$ is also countable. Less immediate topological properties of indicating sets are given below. The proof of the first lemma follows from standard results on rational functions for which we refer to [2].

Lemma 5.3. Let $X=F^{-1}(\widehat{\mathbf{R}})$ for a rational function $F: \widehat{\mathbf{C}} \rightarrow \widehat{\mathbf{C}}$.

(1) Each connected component of $X$ is path connected in $\widehat{\mathbf{C}}$.

(2) If $C$ is any connected component of $X$, then $F(C)=\widehat{\mathbf{R}}$.

Proposition 5.4. Let $G$ be a cocompact Fuchsian group and suppose that $X=$ $R^{-1}(\widehat{\mathbf{R}})$ for a rational map $R$ as defined by (5.4). Then, $X$ is path connected.

Proof. Throughout this proof, we work in the upper-half plane model of $\mathbf{H}$. Let $X=R^{-1}(\widehat{\mathbf{R}})$ where $R$ is given by,

$$
R(z)=\frac{\left(z-f_{2}(z)\right)\left(f_{1}(z)-f_{3}(z)\right)}{\left(z-f_{1}(z)\right)\left(f_{2}(z)-f_{3}(z)\right)}
$$

for distinct, non-identity maps $f_{1}, f_{2}$, and $f_{3} \in G$. For $j \in \mathbf{N}$, let $C_{j}$ denote the connected components of $X$. We begin by showing that $X$ has only one connected component. 
Firstly, observe that $\widehat{\mathbf{R}} \subset X$ : if $z \in \widehat{\mathbf{R}}$, then $R(z) \in \widehat{\mathbf{R}}$ since each $f_{i}$ is a hyperbolic isometry of $\mathbf{H}$ and, hence, fixes $\partial \mathbf{H}=\widehat{\mathbf{R}}$ set-wise. The set $\widehat{\mathbf{R}}$ is connected, and therefore belongs to a single connected component of $X$. Next, we argue that $R^{-1}(\infty) \subset \widehat{\mathbf{R}} \subset X$. Notice that $R(w)=\infty$ if and only if either (1) $\left(w-f_{2}(w)\right)\left(f_{1}(w)-f_{3}(w)\right)=\infty$ or $(2)\left(w-f_{1}(w)\right)\left(f_{2}(w)-f_{3}(w)\right)=0$. We proceed to show that both cases imply that $w \in \widehat{\mathbf{R}}$.

In case $(1)$, either $\left(w-f_{2}(w)\right)=\infty$ or $\left(f_{1}(w)-f_{3}(w)\right)=\infty$ implying that at least one of the following values equals $\infty$ : $w, f_{1}(w), f_{2}(w)$, or $f_{3}(w)$. Since $\infty \in \widehat{\mathbf{R}}$ and each $f_{i}$ fixes $\widehat{\mathbf{R}}$ set-wise, it must be the case that $w \in \widehat{\mathbf{R}}$. In case (2), $w$ satisfies $\left(w-f_{1}(w)\right)\left(f_{2}(w)-f_{3}(w)\right)=0$ implying that either $\left(w-f_{1}(w)\right)=0$ or $\left(f_{2}(w)-f_{3}(w)\right)=0$. It follows that either $w=f_{1}(w)$ or $w=f_{2}^{-1}\left(f_{3}(w)\right)$. Since the hyperbolic isometries $f_{1}$ and $f_{2}^{-1} f_{3}$ only fix points in $\widehat{\mathbf{R}}$, it follows that $w \in \widehat{\mathbf{R}}$ in this case as well.

By (2) of Lemma 5.3, each component $C_{j}$ of $X$ contains a pre-image of $\infty$ under $R$. The argument above now implies that each $C_{j}$ intersects $\widehat{\mathbf{R}}$ non-trivially. Hence, each $C_{j}$ is contained in the single connected component containing $\widehat{\mathbf{R}}$. Thus, $X$ has a single connected component and is path connected by (1) of Lemma 5.3.

The arguments used in the proof above also yield the following corollary when we identify $\mathbf{H}$ with the upper-half plane model.

Corollary 5.5. If $R: \widehat{\mathbf{C}} \rightarrow \widehat{\mathbf{C}}$ is a rational function as defined in (5.4), then $R^{-1}(\infty) \subset \widehat{\mathbf{R}}$.

Suppose $z_{0}$ is exceptional for $G$ and belongs to the indicating set $\Gamma=R^{-1}(\widehat{\mathbf{R}}) \cap \mathbf{H}$. Since $R^{-1}(\widehat{\mathbf{R}})$ is connected and contains $\widehat{\mathbf{R}}, \Gamma$ must be unbounded in the hyperbolic metric. Therefore,

Proposition 5.6. Let $G$ be a cocompact Fuchsian group with exceptional point $z_{0}$ lying on an indicating set $\Gamma$ for $G$. For any $M>0$, there exists a point $z_{M} \in \Gamma$ with $\rho\left(z_{0}, z_{M}\right)>M$ and a path $\gamma:[0,1] \rightarrow \Gamma$ such that $\gamma(0)=z_{0}$ and $\gamma(1)=z_{M}$.

\section{Higher order exceptional points}

Let $G$ be a cocompact Fuchsian group with Dirichlet region $D(z)$ centered at $z \in \mathbf{H}$. Since $G$ is cocompact, the number of sides of $D(z)$, denoted by $|S(z)|$, is even; see [1]. Recall that $|S(z)|=12 g-6$ unless $z$ is exceptional and so it follows that, if $z$ is exceptional, then $S(z) \leq 12 g-8$.

Definition 6.1. If $z$ is an exceptional point for $G$ satisfying $|S(z)| \leq 12 g-10$, then $z$ is called an exceptional point of higher order for $G$.

The next proposition is contained in [5, Theorem 2.2], where its proof is omitted. For convenience, we sketch its proof below.

Proposition 6.2. Let $G$ be a cocompact Fuchsian group. If $z \in \mathbf{H}$ is exceptional but not exceptional of higher order for $G$, then there exists an indicating set $\Gamma$ with $z \in \Gamma$ and an open set $U$ containing $z$ such that $S(w)=S(z)$ for all $w \in U \cap \Gamma$.

Sketch of proof. Let $z \in \mathbf{H}$ be exceptional for $G$, but not exceptional of higher order for $G$. Hence, $S(z)=12 g-8$ and it follows that $D(z)$ has one vertex cycle of length 4 and all other vertex cycles of length 3 ; see the proof of [1, Theorem 10.5.1]. 
Let $C$ denote the boundary cycle of $D(z)$ of length 4 given by $C=\left\{v, f_{1}^{-1}(v), f_{2}^{-1}(v)\right.$, $\left.f_{3}^{-1}(v)\right\}$ for distinct, non-identity maps $f_{1}^{-1}, f_{2}^{-1}$, and $f_{3}^{-1} \in G$ and $v \in \partial D(z)$. The exceptional point $z$ lies on the indicating set $\Gamma=R^{-1}(\widehat{\mathbf{R}}) \cap \mathbf{H}$ where $R(z)$ is given by,

$$
R(z)=\frac{\left(z-f_{2}(z)\right)\left(f_{1}(z)-f_{3}(z)\right)}{\left(z-f_{1}(z)\right)\left(f_{2}(z)-f_{3}(z)\right)}
$$

as in equation (5.4) of Proposition 5.1. In addition, the collection of points $\left\{z, f_{1}(z)\right.$, $\left.f_{2}(z), f_{3}(z)\right\}$ lies on a (hyperbolic) circle in $\mathbf{H}$ centered at $v$ and so, $v$ is the unique point in $\mathbf{H}$ satisfying

$$
\rho(v, z)=\rho\left(v, f_{1}(z)\right)=\rho\left(v, f_{2}(z)\right)=\rho\left(v, f_{3}(z)\right)
$$

for the collection $\left\{z, f_{1}(z), f_{2}(z), f_{3}(z)\right\}$.

It now suffices to prove the following statement: if $\left\{z_{n}\right\} \subset \Gamma$ is any sequence converging to $z$, then $S(z)=S\left(z_{n}\right)$ for large enough $n$. Let $\left\{z_{n}\right\} \subset \Gamma$ be any sequence converging to $z$. By Theorem 3.1, if $n$ is large enough, then $S(z) \subset S\left(z_{n}\right)$. For these large $n$,

$$
12 g-8=|S(z)| \leq\left|S\left(z_{n}\right)\right| \leq 12 g-6 .
$$

It now follows that, if $\left|S\left(z_{n}\right)\right|<12 g-6$, then $S(z)=S\left(z_{n}\right)$ for these $n$. Therefore, we seek to show that $z_{n}$ is exceptional for sufficiently large $n$.

For each $n, z_{n} \notin \widehat{\mathbf{R}}$ and so, by Corollary 5.5 the point $R\left(z_{n}\right) \in \mathbf{R}$. Equivalently, the complex cross-ration $\left[z_{n}, f_{1}\left(z_{n}\right), f_{2}\left(z_{n}\right), f_{3}\left(z_{n}\right)\right]$ is purely real and the points $z_{n}$, $f_{1}\left(z_{n}\right), f_{2}\left(z_{n}\right)$, and $f_{3}\left(z_{n}\right)$ lie on a hyperbolic circle in $\mathbf{H}$. We denote the hyperbolic center of this circle by $v_{n}$. Observe that, for each $n, v_{n}$ is the unique point in $\mathbf{H}$ satisfying

$$
\rho\left(v_{n}, z_{n}\right)=\rho\left(v_{n}, f_{1}\left(z_{n}\right)\right)=\rho\left(v_{n}, f_{2}\left(z_{n}\right)\right)=\rho\left(v_{n}, f_{3}\left(z_{n}\right)\right) .
$$

The $z_{n}$ converge to $z$ by assumption and, hence, for each $j \in\{1,2,3\}, f_{j}\left(z_{n}\right) \rightarrow f_{j}(z)$ as $n \rightarrow \infty$. One now checks that the circles defined by the collection $\left\{z_{n}, f_{1}\left(z_{n}\right)\right.$, $\left.f_{2}\left(z_{n}\right), f_{3}\left(z_{n}\right)\right\}$ converge in the Hausdorff topology to the circle defined by $\left\{z, f_{1}(z)\right.$, $\left.f_{2}(z), f_{3}(z)\right\}$. It follows from (6.1), (6.3), and the continuity of $\rho$, that $v_{n} \rightarrow v \in$ $\partial D(z)$ as defined by (6.1).

Using the discreteness of $G$ and the continuity of $\rho$, it can now be shown that $v_{n} \in \partial D\left(z_{n}\right)$ for large enough values of $n$. However, $\rho\left(z_{n}, f_{j}^{-1}\left(v_{n}\right)\right)=\rho\left(z_{n}, v_{n}\right)$ for $j \in\{1,2,3\}$ by (6.3). So, $\left\{z_{n}, f_{1}^{-1}\left(v_{n}\right), f_{2}^{-1}\left(v_{n}\right), f_{3}^{-1}\left(v_{n}\right)\right\} \subset \partial D\left(z_{n}\right)$ for large enough vales of $n$ and, hence, $\partial D\left(z_{n}\right)$ has at least one boundary cycle of length greater than 3. This implies that $z_{n}$ is exceptional for large enough values of $n$ and completes the proof.

Proposition 6.2 provides a result on exceptional points for $G$ which are not of higher order; its content mirrors that of Propsition 3.3 pertaining to regular points. Proving a comparable result for exceptional points of higher order, however, appears to be more complicated. The methods used thus far fail when considering such points, because these points can admit boundary cycles of various lengths. Still, we can use Proposition 6.2 to establish that higher order exceptioanal points for $G$ always exist.

Theorem 6.3. If $G$ is a cocompact Fuchsian group, then there exists an exceptional point of higher order for $G$. 
Proof. Choose, by Theorem 4.3, an exceptional point $z_{0}$ for $G$ in $\mathbf{H}$. If $z_{0}$ is an exceptional point of higher order, then we are done. So, assume that $z_{0}$ is exceptional for $G$, but not exceptional of higher order for $G$. By Proposition 6.2, there exists an indicating set $\Gamma$ and an open set $U$ containing $z_{0}$ such that $S(w)=S\left(z_{0}\right)$ for all $w \in U \cap \Gamma$. The set $\left\{z \in \mathbf{H}: S(z)=S\left(z_{0}\right)\right\}$ is bounded by Theorem 4.2. Hence, there exists some $M>0$ such that $S(z) \neq S\left(z_{0}\right)$ for all $z$ satisfying $\rho\left(z_{0}, z\right)>M$. Now, apply Proposition 5.6: let $z_{M} \in \Gamma$ with $\rho\left(z_{0}, z_{M}\right)>M$ and let $\gamma:[0,1] \rightarrow \Gamma$ satisfy $\gamma(0)=z_{0}$ and $\gamma(1)=z_{M}$.

Define $x_{0}=\inf \left\{x \in[0,1]: S(\gamma(x)) \neq S\left(z_{0}\right)\right\}$. The properties of the open set $U$, the point $z_{M}$, and the curve $\gamma$ ensure that $0<x_{0} \leq 1$. We claim that $\gamma\left(x_{0}\right) \in \mathbf{H}$ is an exceptional point of higher order for $G$.

Construct an increasing sequence $\left\{x_{n}\right\}_{n \in N} \subset\left[0, x_{0}\right)$ such that $x_{n} \rightarrow x_{0}$ as $n \rightarrow \infty$. Note that the continuity of $\gamma$ implies that $\gamma\left(x_{n}\right) \rightarrow \gamma\left(x_{0}\right)$ as $n \rightarrow \infty$. For all $n \in \mathbf{N}$, $S\left(\gamma\left(x_{n}\right)\right)=S\left(z_{0}\right)$; this follows from the definition of $x_{0}$. By Theorem 3.1,

$$
S\left(\gamma\left(x_{0}\right)\right) \subset S\left(\gamma\left(x_{n}\right)\right)=S\left(z_{0}\right),
$$

for all $n \in \mathbf{N}$. Since $S\left(\gamma\left(x_{0}\right)\right) \neq S\left(z_{0}\right)$ by definition and since $z_{0}$ was chosen to be exceptional for $G$, we have that $\left|S\left(\gamma\left(x_{0}\right)\right)\right| \supsetneqq\left|S\left(z_{0}\right)\right|=12 g-8$. That is, $\left|S\left(\gamma\left(x_{0}\right)\right)\right| \leq$ $12 g-10$ and the proof is complete.

In comparison to Theorem 4.3, the above fact does not establish the existence of uncountably many exceptional points of higher order for $G$. It is easy to see, however, that infinitely many such points exist for $G$ : $G$ is countably infinite and all points in a single $G$-orbit have isometric Dirichlet regions [1]. Unfortunately, there are only countably many indicating sets for $G$. Hence, the methods used to prove Theorem 6.3 cannot produce a result comparable to Theorem 4.3 for exceptional points of higher order collapse.

Acknowledgements. The above results were included in the author's doctoral dissertation completed at Wesleyan University. The author wishes to express deep gratitude to his $\mathrm{PhD}$ adviser, Petra Bonfert-Taylor, for her help and guidance in the completion of this publication. Many thanks, as well, to the referee for their helpful comments and suggestions.

\section{References}

[1] Beardon, A. F.: The geometry of discrete groups. - Grad. Texts in Math. 91, Springer-Verlag, New York, 1983.

[2] Beardon, A. F.: Iteration of rational functions. - Grad. Texts in Math. 132, Springer-Verlag, New York, 1991.

[3] Katok, S.: Fuchsian groups. - Chicago Lectures in Mathematics, University of Chicago Press, Chicago, IL, 1992.

[4] Lehto, O.: Univalent functions on Teichmüller spaces. - Grad. Texts in Math. 109, SpringerVerlag, New York, 1987.

[5] NÄÄtÄnen, M.: On the stability of identification patterns for Dirichlet regions. - Ann. Acad. Sci. Fenn. Ser. A I Math. 10, 1985, 411-417. 